Louisiana State University

LSU Digital Commons

7-1-2005

\title{
V-ATPase inhibition prevents recovery from anoxia in Artemia franciscana embryos: Quiescence signaling through dissipation of proton gradients
}

Joseph A. Covi

Louisiana State University

W. Dale Treleaven

Louisiana State University

Steven C. Hand

Louisiana State University

Follow this and additional works at: https://digitalcommons.Isu.edu/biosci_pubs

\section{Recommended Citation}

Covi, J., Treleaven, W., \& Hand, S. (2005). V-ATPase inhibition prevents recovery from anoxia in Artemia franciscana embryos: Quiescence signaling through dissipation of proton gradients. Journal of Experimental Biology, 208 (14), 2799-2808. https://doi.org/10.1242/jeb.01681

This Article is brought to you for free and open access by the Department of Biological Sciences at LSU Digital Commons. It has been accepted for inclusion in Faculty Publications by an authorized administrator of LSU Digital Commons. For more information, please contact ir@lsu.edu. 


\section{Erratum}

Covi, J. A., Treleaven, W. D. and Hand, S. C. (2005). V-ATPase inhibition prevents recovery from anoxia in Artemia franciscana embryos: quiescence signaling through dissipation of proton gradients. J. Exp. Biol. 208, 2799-2808.

In the final print version and the pdf version available on-line, an incomplete version of Fig. 6 was published, with the lower $x$ axis and labels obscured by the figure legend. The full-text version on-line is correct. The complete figure is reproduced below.
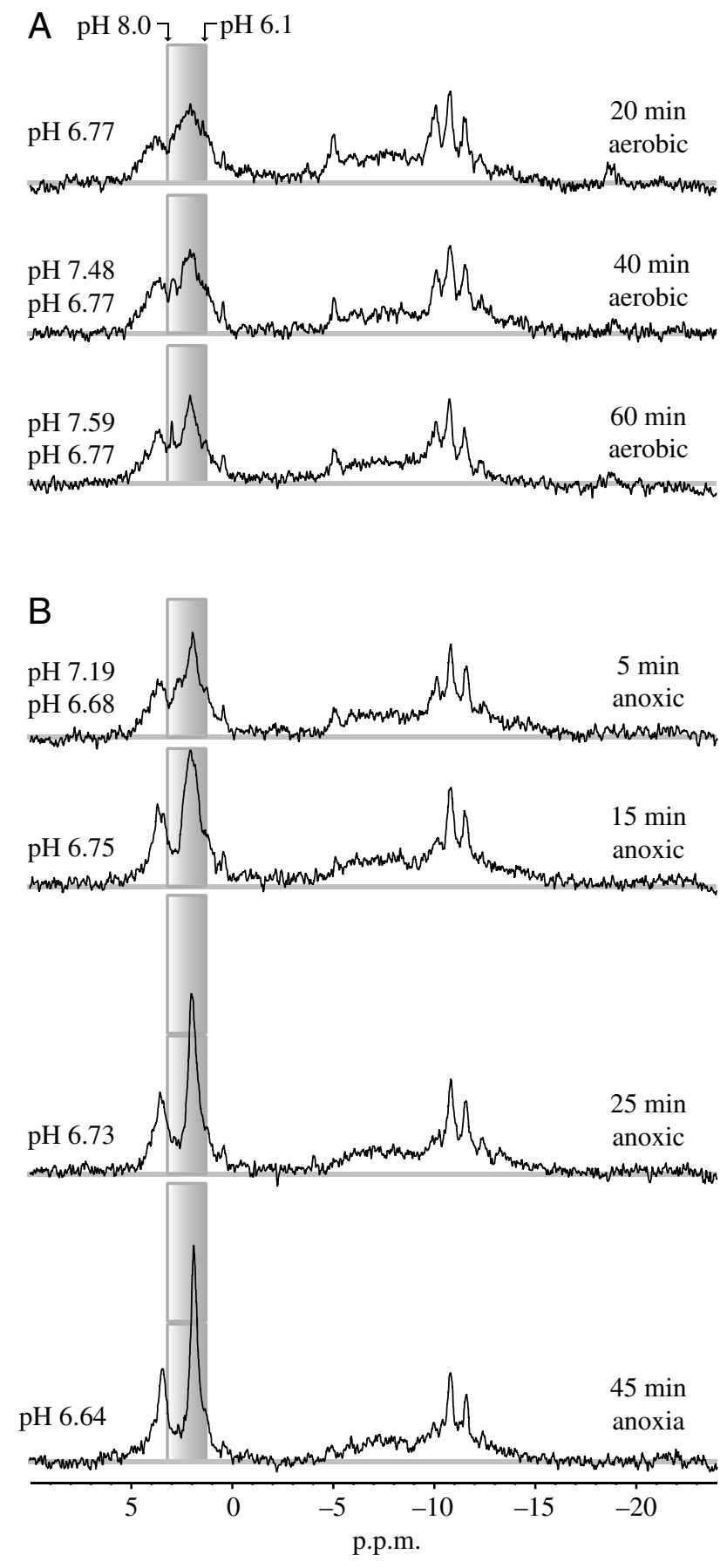

We apologise for any inconvenience this may have caused readers. 
doi: $10.1242 /$ jeb. 01681

\title{
V-ATPase inhibition prevents recovery from anoxia in Artemia franciscana embryos: quiescence signaling through dissipation of proton gradients
}

\author{
Joseph A. Covi ${ }^{1, *}$, W. Dale Treleaven ${ }^{2}$ and Steven C. Hand ${ }^{1}$ \\ ${ }^{1}$ Division of Cellular, Developmental and Integrative Biology, Department of Biological Science and ${ }^{2}$ NMR Facility, \\ College of Basic Sciences, Louisiana State University, Baton Rouge, LA 70803, USA
}

*Author for correspondence (e-mail: jcovi1@1su.edu)

Accepted 11 May 2005

\begin{abstract}
Summary
The metabolic downregulation critical for long-term survival of Artemia franciscana embryos under anoxia is mediated, in part, by a progressive intracellular acidification. However, very little is known about the mechanisms responsible for the $\mathrm{pH}$ transitions associated with exposure to, and recovery from, oxygen deprivation. In the present study, we demonstrate with ${ }^{31}$ P-NMR that incubation of intact embryos with the V-ATPase inhibitor bafilomycin $A_{1}$ severely limits intracellular alkalinization during recovery from anoxia without affecting the restoration of cellular nucleotide triphosphate levels. Based on these data, it appears that oxidative phosphorylation and ATP resynthesis can only account for the first $0.3 \mathrm{pH}$ unit alkalinization observed during aerobic recovery from the $1 \mathrm{pH}$ unit acidification produced during $1 \mathrm{~h}$ of anoxia. The additional $0.7 \mathrm{pH}$ unit increase requires proton pumping by the V-ATPase. Aerobic incubation with bafilomycin also suggests that $\mathrm{V}$ -

ATPase inhibition alone is not enough to induce an acute dissipation of proton gradients under anoxia. In intact embryos, the dissipation of proton gradients and uncoupling of oxidative phosphorylation with carbonyl cyanide 3-chlorophenylhydrazone (CCCP) leads to an intracellular acidification similar to that seen after $1 \mathrm{~h}$ of anoxia. Subsequent exposure to anoxia, in the continued presence of CCCP, yields little additional acidification, suggesting that proton gradients are normally dissipated under anoxia. When combined with protons generated from net ATP hydrolysis, these data show that the dissipation of proton chemical gradients is sufficient to account for the reversible acidification associated with quiescence in these embryos.

Key words: vacuolar-type ATPase, CCCP, bafilomycin $\mathrm{A}_{1}$, quiescence, gradient dissipation, anoxia, acidification, intracellular $\mathrm{pH},{ }^{31} \mathrm{P}-\mathrm{NMR}$.
\end{abstract}

\section{Introduction}

For more than 20 years, the source of the impressive intracellular acidification induced by anoxia in encysted embryos of the brine shrimp, Artemia franciscana, has eluded a complete explanation. In the preceding companion paper (Covi and Hand, 2005), we proposed a novel mechanism for regulated acidification of the intracellular space, in which exposure to anoxia would trigger a net flux of protons from acidic compartments to the surrounding cytoplasm. Given the rapid drop in cellular ATP:ADP ratio occurring under anoxia in these embryos (Anchordoguy and Hand, 1994; Stocco et al., 1972), this process would probably involve inactivation of ATP-dependent proton pumping into these compartments (cf. Huss and Wieczorek, 2003; Kettner et al., 2003). Here we demonstrate that in vivo inhibition of V-ATPase activity in encysted embryos both induces a reversible intracellular acidification under aerobic conditions and precludes recovery from the intracellular acidification induced by anoxia. In addition, we show that global dissipation of proton gradients with carbonyl cyanide 3-chlorophenylhydrazone (CCCP) yields an acidic intracellular $\mathrm{pH}\left(\mathrm{pH}_{\mathrm{i}}\right)$ similar to that observed after $1 \mathrm{~h}$ of anoxia. We argue that, together, these data offer strong support for the hypothesis that proton gradient dissipation is a key contributor to anoxia-induced acidification of the intracellular space in A. franciscana embryos. Indeed, when combined with the protons produced by net hydrolysis of ATP (Busa and Nuccitelli, 1984; Kwast et al., 1995), the collapse of proton chemical gradients can fully explain the origin of acid equivalents required for this acidification.

Descriptive observations of Artemia development can be traced to the early 1900s (for review, see Clegg and Conte, 1980), and extensive investigation of the mechanisms involved in the extreme anoxia tolerance displayed by the encysted embryonic stage continues on many fronts (e.g. Clegg et al., 2000; Eads and Hand, 2003; Menze et al., 2005; Warner et al., 2002; Willsie and Clegg, 2002). However, until now, functional examination of these mechanisms in vivo has been hampered by the presence of a cyst shell that encapsulates these embryos. This shell can be divided into two main 
sections: tertiary envelope (chorion) and embryonic cuticle (Drinkwater and Clegg, 1991), which together present a physical barrier conferring impermeability to all but lowmolecular-mass gasses and water (Trotman, 1991). It even appears to prevent the passage of protons (Busa et al., 1982) and volatile solvents such as DMSO (Price, 1967). In our companion paper, however, we demonstrated that removal of the chorion confers permeability to macrolide antibiotics such as bafilomycin $\mathrm{A}_{1}$, a highly specific and lipid-soluble inhibitor of the V-ATPase (Covi and Hand, 2005). This method of permeabilization is used advantageously in the present study and promises to expand the experimental repertoire available for other in vivo experiments in the future with these encysted embryos.

The V-ATPase is a proton pump that is well known for its nearly ubiquitous role in the acidification of intracellular compartments (Forgac, 2000; Futai et al., 2000). These compartments, diagrammed in Fig. 1, include early and late endosomes, Golgi complex, exocytotic and protein trafficking vesicles, lysosomes and yolk platelets (Fagotto, 1995; Nishi and Forgac, 2002). Not surprisingly, a large number of physiological processes depend on V-ATPase activity, including endocytosis, exocytosis, protein targeting, receptor recycling and lysosomal function (Nishi and Forgac, 2002). Of particular relevance when considering A. franciscana embryos is the finding that the acidification of yolk platelets for degradation requires an ATP-dependent proton pump in insects (Abreu et al., 2004) and is inhibited by bafilomycin in Xenopus

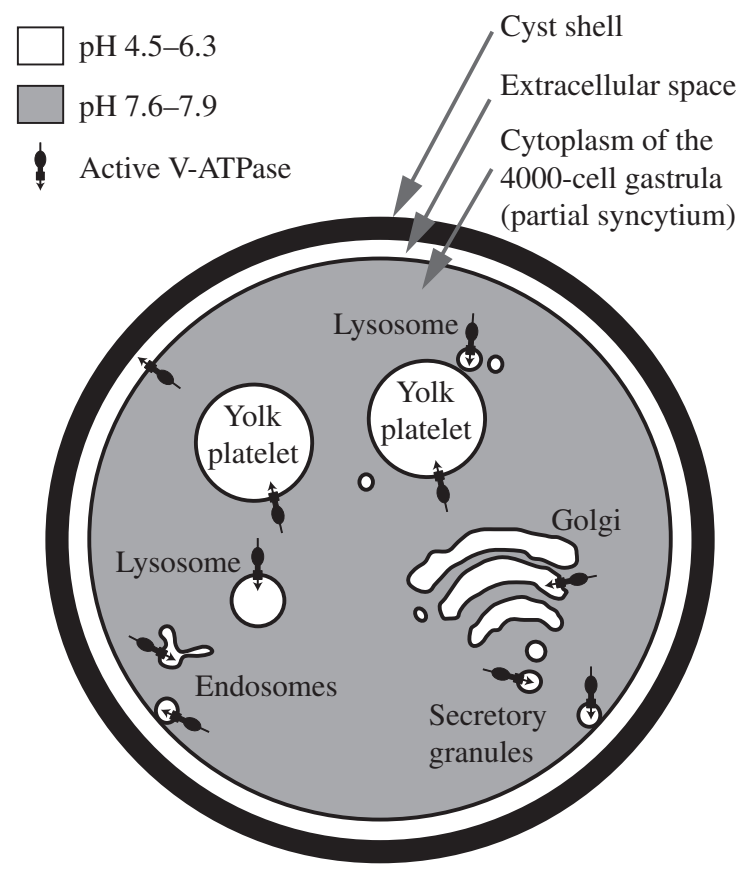

Fig. 1. Diagram describing the potential acidic compartments within a developing encysted gastrula of the brine shrimp, Artemia franciscana. Degree of acidification is indicated by grey scale. Maintenance of steady-state $\mathrm{pH}$ within acidic compartments is assumed to be regulated largely by ATP-dependent proton pumping, ion leak rates and buffering capacity. oocytes (Fagotto and Maxfield, 1994) and Rhodnius prolixus eggs (Motta et al., 2004). The only form of regulation of VATPase activity known to have physiological relevance is the dissociation of the peripheral $\left(\mathrm{V}_{1}\right)$ sector from the membranespanning $\left(\mathrm{V}_{0}\right)$ domain (Wieczorek et al., 2003), and this disassembly may be a mechanism for inactivating the VATPase when energy availability is limited (Kane and Smardon, 2003).

The ability to survive prolonged bouts of anoxia is directly linked to the depression of cellular energy turnover (Hand, 1998). This correlate is exemplified in A. franciscana embryos, for which it has reasonably been argued that long-term survivorship under anoxia is accomplished in part through the establishment of a nearly ametabolic state (Clegg, 1997; Warner and Clegg, 2001). We previously proposed that the drop in cellular ATP:ADP ratio and increase in free inorganic phosphate $\left(\mathrm{P}_{\mathrm{i}}\right)$ are likely to inactivate the V-ATPase within minutes of exposing these embryos to anoxia and that subsequent dissipation of proton chemical gradients could result in a substantial cytoplasmic acidification (Covi and Hand, 2005). Here, we use the technique of phosphorous nuclear magnetic resonance $\left({ }^{31} \mathrm{P}-\mathrm{NMR}\right)$ to directly test this hypothesis by observing the effects of V-ATPase inhibition and proton gradient dissipation on the $\mathrm{pH}_{\mathrm{i}}$ status of intact brine shrimp embryos. The protonophore CCCP is also used to examine the effects of complete dissipation of cellular proton gradients. Specifically, this lipid-soluble weak acid facilitates a comparison of intracellular acidification under anoxia with the acidification induced by combining the uncoupling of oxidative phosphorylation with the dissipation of proton gradients.

\section{Materials and methods Materials}

Instant Ocean was purchased from Aquarium Systems (Eastlake, OH, USA). Gentamycin sulfate and CCCP were obtained from Sigma (St Louis, MO, USA). Bafilomycin $\mathrm{A}_{1}$ (also referred to as bafilomycin) was either obtained from AG Scientific (San Diego, CA, USA) or LC Laboratories (Woburn, MA, USA). Both bafilomycin and CCCP were dissolved in $100 \%$ ethanol, and stock solutions were stored at $-20^{\circ} \mathrm{C}$. Low endotoxin sucrose for ${ }^{31} \mathrm{P}-\mathrm{NMR}$ and respirometry experiments was obtained from Pfanstiehl (Waukegan, IL, USA). All other chemicals used were of the highest grade available.

\section{Animals}

Encysted embryos of the brine shrimp Artemia franciscana Kellog (Great Salt Lake population) were obtained in the dehydrated state (post-diapause) from Sanders Brine Shrimp Co. (Ogden, UT, USA) in 2001. These post-diapause embryos were stored dry at $-20^{\circ} \mathrm{C}$ and hydrated overnight at $0^{\circ} \mathrm{C}$ in $0.25 \mathrm{~mol} \mathrm{l}^{-1} \mathrm{NaCl}$ prior to dechorionation or aseptic washing. Dechorionation was performed as previously described (Kwast and Hand, 1993). Aseptic washing was performed using the 
same protocol, but the antiformin $(\mathrm{NaOH} /$ hypochlorite solution) wash was kept at $0^{\circ} \mathrm{C}$ to prevent complete removal of the chorion. Aseptically treated embryos were used in the $16 \mathrm{~h}$ anoxia study only. Dechorionated embryos were used in all other experiments. Embryos were stored for no more than $16 \mathrm{~h}$ in ice cold $0.125 \mathrm{~mol}^{-1} \mathrm{NaCl}$ prior to experimentation. All solutions were prepared less than $24 \mathrm{~h}$ prior to use, and the length of incubation time for embryos in antiformin was strictly maintained at $20 \pm 1 \mathrm{~min}$ at room temperature. Developmental staging of embryos was conducted using the same criteria described by Conte et al. (1977).

\section{NMR spectroscopy}

Proton-decoupled ${ }^{31} \mathrm{P}$-NMR spectra were collected at a frequency of $202.458 \mathrm{MHz}$ at a temperature of $298 \pm 0.1 \mathrm{~K}$ using a Brucker (Bellerica, MA, USA) AMX-500 spectrometer. 2048 data points were collected over a spectral width of 82 p.p.m., and datasets were zero-filled to $32 \mathrm{~K}$. Except during the first hour of anoxia and recovery from anoxia, 3072 transients were recorded over a period of $10 \mathrm{~min}$ in each experiment. During these transitions between aerobic and anoxic conditions, 1536 transients were recorded over $5 \mathrm{~min}$ in order to provide better time resolution of $\mathrm{pH}$ shifts. Data were exponentially multiplied by $10 \mathrm{~Hz}$ line-broadening prior to Fourier transformation. Zero reference was established using a $85 \% \mathrm{H}_{3} \mathrm{PO}_{4}$ standard. Field drift was assessed by the acquisition of $85 \% \mathrm{H}_{3} \mathrm{PO}_{4}$ spectra before and after each experiment. A deuterium lock was not required, as field drift in our system $\left(0.057 \mathrm{~Hz} \mathrm{~h}^{-1}\right)$ was much lower than resolution $\left(0.4 \mathrm{~Hz}\right.$ data point $\left.{ }^{-1}\right)$.

Embryos were packed by gravity into a $10 \mathrm{~mm}$ glass NMR tube, and movement during perfusion was prevented by placing a foam plug at the top of the embryo column. For bafilomycin treatments and controls, embryos were superfused from the bottom of the tube with $0.2 \mathrm{~mol}^{-1}$ sucrose. For CCCP treatment and $16 \mathrm{~h}$ anoxia study, embryos were superfused with $0.25 \mathrm{~mol} \mathrm{l}^{-1} \mathrm{NaCl}$. It should be noted that control spectra obtained using sucrose superfusion were indiscernible from those obtained with $\mathrm{NaCl}$ superfusion (data not shown). Both sucrose and $\mathrm{NaCl}$ solutions were sterile filtered before use, and the final concentration of ethanol during treatments was $0.2 \%$. Maintenance of aerobic conditions and induction of anoxia during in vivo spectroscopy were conducted as described by Kwast et al. (1995). In brief, embryos were superfused with oxygen saturated medium at a rate of $2 \mathrm{ml} \mathrm{min}^{-1}$, and anoxia was induced by stopping perfusion. As discussed by Kwast et al. (1995), the use of oxygen-saturated medium has no noticeable affect on embryonic development, and stopping superfusion provides the most rapid production of anoxic conditions. Peak identification was accomplished by comparison with published spectra (Busa et al., 1982; Kwast et al., 1995). Intracellular pH was estimated from the chemical shift (resonance frequency) of $\mathrm{P}_{\mathrm{i}}$ using a titration curve generated in our facility (Fig. 2C). This standard curve was produced using a solution of $10 \mathrm{mmol} \mathrm{l}^{-1} \mathrm{P}_{\mathrm{i}}$, $110 \mathrm{mmol} \mathrm{l}^{-1} \mathrm{KCl}$ and $20 \mathrm{mmol} \mathrm{l}^{-1} \mathrm{NaCl}$, and $\mathrm{pH}$ was set by the combination of mono- and dibasic phosphate.
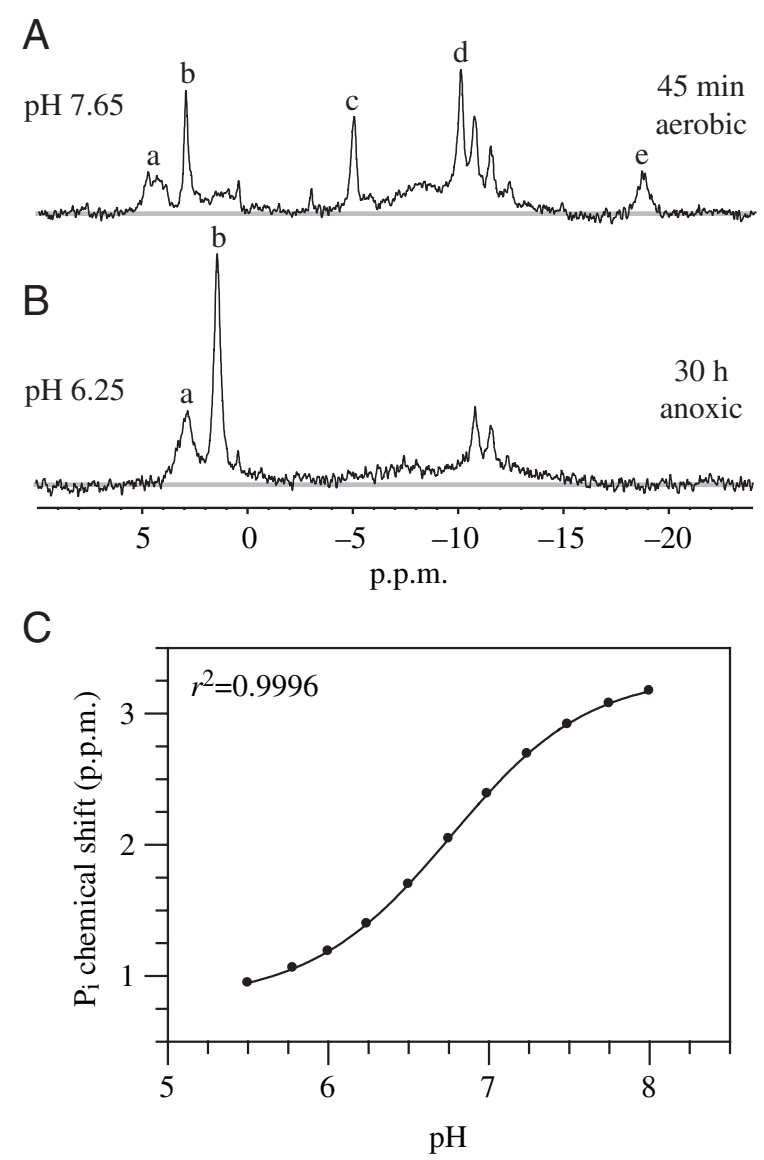

Fig. 2. Representative ${ }^{31} \mathrm{P}-\mathrm{NMR}$ spectra of intact A. franciscana embryos collected after (A) 45 min of aerobic development or (B) $30 \mathrm{~h}$ of anoxia following $1 \mathrm{~h}$ of aerobic development. Duration and nature of treatments are listed to the right of each spectrum. Average embryonic (intracellular) $\mathrm{pH}$ is listed to the left. Predominant chemical shifts for phosphate nuclei are identified by letters: a, phosphomonoesters; $b$, inorganic phosphate $\left(\mathrm{P}_{\mathrm{i}}\right)$; $\mathrm{c}, \gamma$-NTP and $\beta$ NDP; d, $\alpha$-NTP and NDP; e, $\beta$-NTP. (C) A standard curve relating the chemical shift of $\mathrm{P}_{\mathrm{i}}(\delta)$ to solution $\mathrm{pH}$ was used to determine the average intracellular $\mathrm{pH}$ during treatments. This curve is described by the equation $\mathrm{pH}=6.76+\log [(\delta-0.82) /(3.31-\delta)]$.

\section{Hatching assays}

Hatching success was observed for embryos used in ${ }^{31} \mathrm{P}$ NMR experiments. To remove inhibitors and ethanol from the incubation medium after NMR measurements were made, embryos were gently resuspended in the NMR tube using 35\%o artificial seawater at room temperature, and care was taken to use only the embryos that were contained within the spectrometer detection window. These embryos were transferred to a Durawipe (Johnson and Johnson Advanced Materials Co., New Brunswick, NJ, USA) filter funnel, rinsed with artificial seawater and resuspended in fresh $35 \%$ artificial seawater for 15-30 min prior to transferring to cell culture plates for hatching tests. Embryos were allowed to develop in the dark for 66-69 $\mathrm{h}$ before counting. This incubation period was sufficient for completion of hatching, as no further 
development was observed in individuals that did not reach the naupliar stage after $66 \mathrm{~h}$. It is relevant to note that recovery of $\mathrm{pHi}$ after removal of bafilomycin was not tested, as it is extremely difficult to remove bafilomycin from A. franciscana embryos. For example, the depressed hatching rates caused by exposure to $4 \mu \mathrm{mol} \mathrm{l}^{-1}$ bafilomycin for $24 \mathrm{~h}$ at $0^{\circ} \mathrm{C}(10 \%$ hatch $)$ are only slightly reversed by 5.5 days of washing in inhibitorfree medium at $0^{\circ} \mathrm{C}\left(21 \%\right.$ hatch). Given the low $K_{\mathrm{D}}$ $\left(10^{-8} \mathrm{~mol} \mathrm{l}^{-1}\right)$ and lipophilic nature of bafilomycin (Drose and Altendorf, 1997), this could be the result of strong binding between inhibitor and enzyme, or a product of slow diffusion out of the lipid-rich cell mass of these embryos.

\section{Respirometry}

Respiration measurements were conducted in a solution of $0.2 \mathrm{~mol} \mathrm{l}^{-1}$ sucrose. CCCP was added $2.75 \mathrm{~h}$ after embryos were placed in respiration medium at $25^{\circ} \mathrm{C}$, and the final ethanol concentration after addition was $0.5 \%$. Respiration medium was sterile filtered prior to use, and oxygen consumption due to any bacterial contamination was limited by the inclusion of $50 \mu \mathrm{g} \mathrm{ml}^{-1}$ gentamycin sulfate. Gentamycin sulfate has no noticeable effect on survivorship of $A$. franciscana embryos (data not shown).

Dechorionated embryos were blotted dry, and 2-3 mg (approximately 230-350 embryos) were weighed directly in a $1 \mathrm{ml}$ Eppendorf tube. Embryos were resuspended in respiration medium within 4-6 min of blotting dry and immediately transferred to a water-jacketed respiration chamber (model RC350; Strathkelvin Instruments, Glasgow, Scotland, UK) that was maintained at $25^{\circ} \mathrm{C}$. Oxygen tension was measured in $1.5 \mathrm{ml}$ of medium using a polarographic oxygen electrode (Strathkelvin model 1302) under room lighting, and data were collected with DataCan V acquisition software from Sable Systems (Las Vegas, NV, USA). Raw data were analyzed with DatGraf software from Cyclobios (Innsbruck, Austria). Oxygen consumption by the electrodes, back diffusion into the system, and electrode time constants were corrected for as previously described (Kwast et al., 1995). Oxygen tension was recorded continuously for $6.5 \mathrm{~h}$ with gentle stirring, and the electrode was raised at regular predetermined intervals to introduce air for gas exchange. In this way, $\mathrm{CO}_{2}$ accumulation was limited, and oxygen tension was prevented from dropping below $65 \%$ air saturation. It is important to note that, while we observed no lysis or homogenization of embryos during respirometry experiments, continuous stirring does appear to increase permeability of the cuticle and decrease subsequent hatching success in controls. Thus, respiration effects observed with stirring cannot be directly compared with ${ }^{31} \mathrm{P}-\mathrm{NMR}$, which has no detrimental effect on hatching rates for control treatments.

\section{Results}

Spectra obtained from aerobic (Fig. 2A) and anoxic (Fig. 2B) A. franciscana embryos were very similar in appearance to those previously published by our lab and others (Busa et al., 1982; Kwast et al., 1995). Average embryonic (intracellular) $\mathrm{pH}$ was estimated using the chemical shift of $\mathrm{P}_{\mathrm{i}}$ and a standard curve relating the $\mathrm{P}_{\mathrm{i}}$ chemical shift to solution $\mathrm{pH}$ (Fig. 2C). For encysted embryos, intracellular $\mathrm{pH}$ after $1 \mathrm{~h}$ of superfusion was estimated by ${ }^{31} \mathrm{P}-\mathrm{NMR}$ to be $7.70 \pm 0.04$ (mean \pm S.E.M.; $N=7$ ). This is slightly lower than the $\mathrm{pH}$ of $\geqslant 7.9$ reported by Busa et al. (1982) but identical to the $7.70 \pm 0.02$ reported by Kwast et al. (1995). During $1 \mathrm{~h}$ of anoxia, intracellular $\mathrm{pH}$ decreased to $6.66 \pm 0.03(N=3)$, and restoration of aerobic superfusion after $1 \mathrm{~h}$ of anoxia produced a rapid alkalinization to $\mathrm{pH} 7.64 \pm 0.05(N=3)$ over a period of $22.5 \mathrm{~min}$. Both of these measurements are also very similar to those reported by Kwast et al. (1995) for $1 \mathrm{~h}$ of anoxia $(6.73 \pm 0.02)$ and $20 \mathrm{~min}$ of aerobic recovery $(7.73 \pm 0.02)$ following $2 \mathrm{~h}$ of anoxia. The lowest $\mathrm{pH}$ we observed was 6.25 , which was recorded after $30 \mathrm{~h}$ of continuous anoxia (Fig. 2B). It is relevant to note that the ${ }^{31} \mathrm{P}-\mathrm{NMR}$ observations made by Kwast et al. (1995) employed aseptically treated embryos (chorion intact) superfused with $0.25 \mathrm{~mol} \mathrm{l}^{-1} \mathrm{NaCl}$, while those discussed above were made using dechorionated embryos superfused with $0.2 \mathrm{~mol} \mathrm{l}^{-1}$ sucrose. This demonstrates that both steady-state $\mathrm{pH}_{\mathrm{i}}$ under aerobic conditions and the transitions in $\mathrm{pH}_{\mathrm{i}}$ associated with exposure to anoxia are insensitive to the surrounding medium even if the chorion is removed.

Aerobic superfusion of encysted embryos produced a single $\mathrm{P}_{\mathrm{i}}$ resonance during the first $1.5 \mathrm{~h}$ of development, after which a second resonance appeared slightly upfield (to the right) of the first (Fig. 3A,B). The $\mathrm{pH}$ indicated by both of these chemical shifts was relatively stable in control embryos until exposure to anoxia (Fig. 3A), upon which they merged and moved progressively upfield in accordance with the predicted intracellular acidification. Relative to the control, exposure to $4 \mu \mathrm{mol} \mathrm{l}^{-1}$ bafilomycin beginning at $1.5 \mathrm{~h}$ of development had no noticeable effect on steady-state $\mathrm{pHi}$, acidification induced by anoxia or alkalinization during aerobic recovery (Fig. 3A). By contrast, when the embryos were incubated with bafilomycin for $24 \mathrm{~h}$ at $0^{\circ} \mathrm{C}$ (metabolism and development blocked by cold) prior to ${ }^{31} \mathrm{P}-\mathrm{NMR}$, steady-state aerobic $\mathrm{pHi}$ was noticeably acidified, and realkalinization of $\mathrm{pHi}$ after anoxia was severely blunted (Fig. 4A). After 20 min of aerobic incubation, a single broad $\mathrm{P}_{\mathrm{i}}$ resonance indicated a slight acidification in embryos pretreated for $24 \mathrm{~h}$ with bafilomycin (Fig. 4A,B). Recovery from this acidification appears to be asynchronous, given that the breadth of the $\mathrm{P}_{\mathrm{i}}$ peak lessens during aerobic incubation (Fig. 4B). Importantly, the three phosphate resonances produced by di- and trinucleotide phosphates (NDP and NTP; primarily ATP, ADP, GTP and GDP) appear to be unaffected by bafilomycin treatment (compare Fig. 4B to Fig. 2A), which indicates that the embryos are indeed viable and metabolically active after the $24 \mathrm{~h}$ treatment with bafilomycin.

Following $1 \mathrm{~h}$ of aerobic superfusion, exposure to $1 \mathrm{~h}$ of anoxia ( $26 \mathrm{~h}$ exposure to bafilomycin) produced a 0.87 unit acidification, which was accompanied by a large reduction in the size of the NTP and NDP chemical shifts (Fig. 4C). This response is very similar to the anoxic response originally 


\section{Dissipation of proton gradients in A. franciscana embryos 2803}

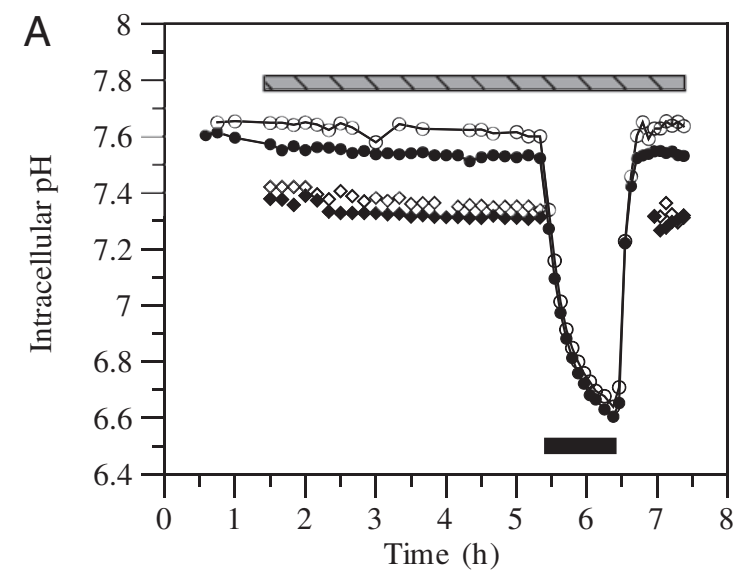

B

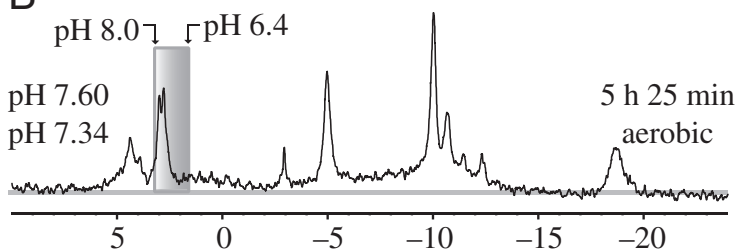

Fig. 3. Intracellular $\mathrm{pH}$ and NTP/NDP status of dechorionated $A$. franciscana embryos during acute treatment with bafilomycin as observed with ${ }^{31} \mathrm{P}-\mathrm{NMR}$. (A) Intracellular $\mathrm{pH}$ is plotted as a function of time for two $\mathrm{P}_{\mathrm{i}}$ chemical shifts identified during early development. Circles represent the downfield (left) $\mathrm{P}_{\mathrm{i}}$ resonance, while diamonds represent the upfield (right) $\mathrm{P}_{\mathrm{i}}$ resonance. Open symbols indicate $4 \mu \mathrm{mol} \mathrm{l}^{-1}$ bafilomycin treatment while filled symbols indicate $0.2 \%$ ethanol control treatment. Period of bafilomycin or ethanol treatment is indicated by a hashed bar, and anoxic period is indicated by a solid bar. (B) Representative spectrum for aerobic embryos, demonstrating the presence of two distinct $P_{i}$ peaks, which appear as a part of normal aerobic development. Two $\mathrm{pH}$ values are given to indicate that a range of $\mathrm{pH}$ is evident from the observed $\mathrm{P}_{\mathrm{i}}$ peaks.

described by Busa et al. (1982), suggesting that acidification mechanisms are still functional after prolonged bafilomycin exposure. By contrast, intracellular alkalinization during aerobic recovery was severely inhibited in embryos exposed to prolonged bafilomycin treatment (Fig. 4A). While an initial rapid alkalinization of $0.3 \mathrm{pH}$ units was observed during the first 17.5 min of aerobic recovery, no further shift in the predominant $\mathrm{P}_{\mathrm{i}}$ resonance was observed. The broad nature of the phosphate peak indicated that heterogeneity of $\mathrm{pH}$ was still present during recovery from anoxia (Fig. 4D). Indeed, $2 \mathrm{~h}$ after the restoration of aerobic superfusion, the $\mathrm{P}_{\mathrm{i}}$ resonance indicated that the average intracellular $\mathrm{pH}$ ranged from 7.19 to 6.72 . It should be noted that we cannot be certain whether this $\mathrm{pH}$ range is entirely the product of heterogeneity among individuals or whether it is also the result of variation in $\mathrm{pH}$ among multiple compartments within individual embryos. It is also important to note that NTP and NDP resonance signatures returned within $17.5 \mathrm{~min}$ of reestablishing aerobic conditions (Fig. 4D), which indicates that oxidative phosphorylation was restored.

The disparity in response observed between acute and prolonged treatments with bafilomycin suggests a time dependency for loading effective quantities of the inhibitor. This possibility is supported by the observation that preincubation of dechorionated embryos with $1 \mu \mathrm{mol} \mathrm{l}^{-1}$ bafilomycin for $24 \mathrm{~h}$ on ice did not impair recovery from anoxia (data not shown), while $4 \mu \mathrm{mol} \mathrm{l}^{-1}$ bafilomycin clearly did. Movement of the inhibitor across the embryonic cuticle may be too slow to effect a change in $\mathrm{pH}$ status during acute treatment.

In addition to macrolide antibiotics, dechorionated embryos of A. franciscana are also permeable to the protonophore CCCP. Incubation with $50 \mu \mathrm{mol}{ }^{-1} \mathrm{CCCP}$ at $25^{\circ} \mathrm{C}$ produced a rapid rise in oxygen consumption by whole embryos (Fig. 5), as would be predicted if the protonophore reached the mitochondrial inner membrane and uncoupled oxidative phosphorylation. This initial increase was followed by prolonged decrease in oxygen consumption consistent with increasing mortality, which is expected to accompany complete uncoupling oxidative phosphorylation (Fig. 5). Preincubation with $20 \mu \mathrm{mol} \mathrm{l}^{-1} \mathrm{CCCP}$ for $30 \mathrm{~h}$ at $0^{\circ} \mathrm{C}$ (metabolism and development blocked by cold) has a large effect on the metabolic status and $\mathrm{pH}_{\mathrm{i}}$ of dechorionated embryos when rewarmed to $25^{\circ} \mathrm{C}$. In these CCCP-treated embryos, the predominant $\mathrm{P}_{\mathrm{i}}$ resonance, even though quite broad, indicates an acidified $\mathrm{pH}_{\mathrm{i}}$ of 6.77 after a brief $20 \mathrm{~min}$ of aerobic incubation (Fig. 6A). Also, as expected from exposure to an uncoupler of oxidative phosphorylation, NTP and NDP resonance peaks are greatly reduced relative to control embryos (Fig. 6A versus Fig. 2A). The broadness of the main $\mathrm{P}_{\mathrm{i}}$ resonance, and presence of a small additional alkaline peak, may indicate differential permeability to $\mathrm{CCCP}$ among embryos. Upon exposure to anoxia, very little shift was observed for the main (acidic) $\mathrm{P}_{\mathrm{i}}$ resonance (Fig. 6B), although the broadness of the peak decreased. Concurrently, the remaining NTP and NDP signals disappeared. Based on the nature of this main $\mathrm{P}_{\mathrm{i}}$ resonance, a key point to be made is that exposure to anoxia induced little additional acidification beyond that already caused by CCCP.

Recovery from the intracellular acidification induced by anoxia is just as rapid after $16.5 \mathrm{~h}$ of exposure as it is after $2 \mathrm{~h}$. Previous observations demonstrated that after $2 \mathrm{~h}$ of anoxia, $\mathrm{pH}_{\mathrm{i}}$ alkalinizes from 6.7 to 7.7 within the first $20 \mathrm{~min}$ of reoxygenation (Kwast et al., 1995). The present study shows that after $16.5 \mathrm{~h}$ of anoxia the restoration of $\mathrm{pH}_{\mathrm{i}}$ to aerobic values also occurs within the first $20 \mathrm{~min}$ of reoxygenation, even though pH dropped to a low of 6.35 under anoxia (Fig. 7). This rapid alkalinization of intracellular $\mathrm{pH}$ stands in contrast with the relatively lengthy time required for full acidification to $\mathrm{pH} 6.35$.

\section{Discussion}

In the companion paper (Covi and Hand, 2005), we demonstrated that dechorionated embryos of the brine shrimp, Artemia franciscana, are permeable to macrolide antibiotics such as bafilomycin and that exposure to sub-micromolar 
A

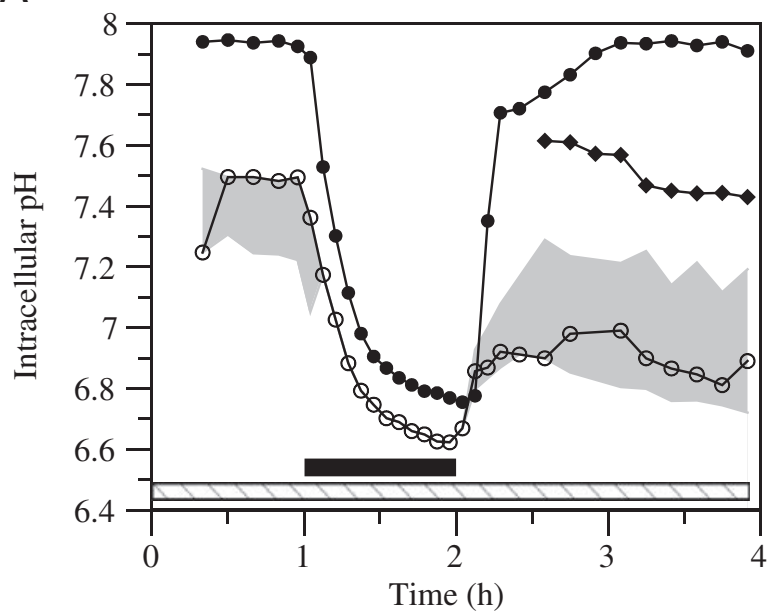

B

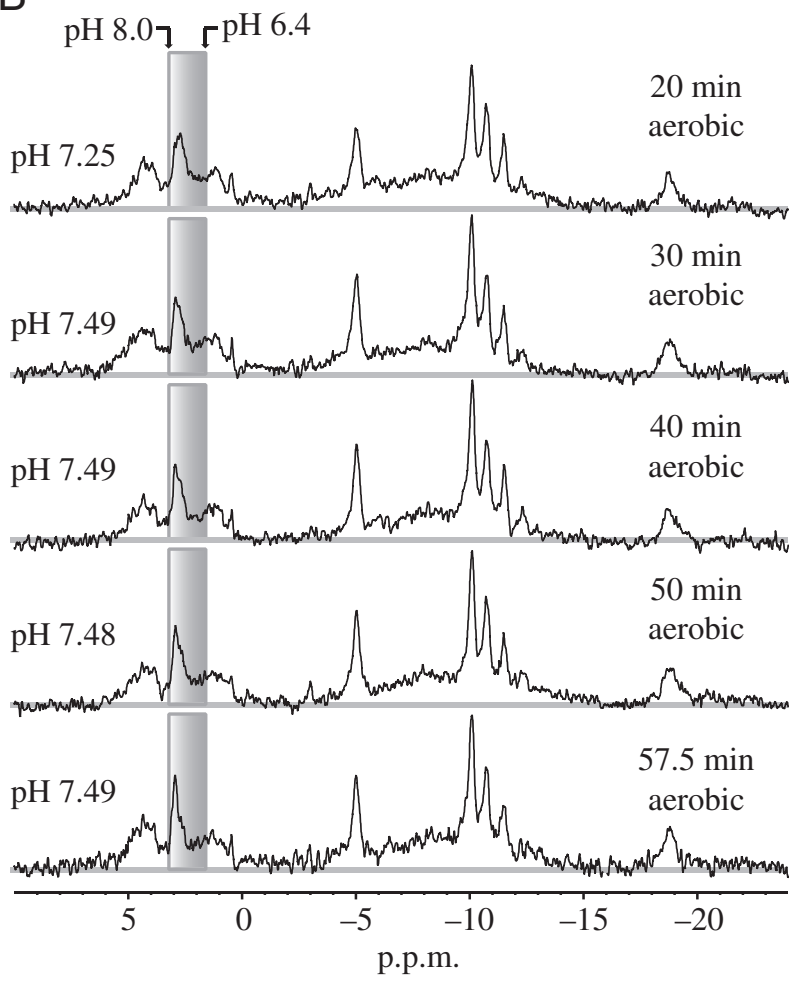

Fig. 4. Intracellular $\mathrm{pH}$ and NTP/NDP status of dechorionated $A$. franciscana embryos for long-term treatment with bafilomycin as observed with ${ }^{31} \mathrm{P}-\mathrm{NMR}$. (A) Intracellular $\mathrm{pH}$ is plotted as a function of time for two $\mathrm{P}_{\mathrm{i}}$ chemical shifts identified during early development. Open circles indicate $\mathrm{pH}$ estimated using the predominant $\mathrm{P}_{\mathrm{i}}$ chemical shifts for embryos pretreated with $4 \mu \mathrm{mol}^{-1}$ bafilomycin on ice for $24 \mathrm{~h}$ prior to acquisition of NMR spectra. Filled circles indicate $\mathrm{pH}$ determined from main downfield $\mathrm{P}_{\mathrm{i}}$ resonance for control embryos given identical treatment but in the absence of bafilomycin. Filled diamonds indicate the upfield (acidic) $\mathrm{P}_{\mathrm{i}}$ resonance for control embryos, which appears after $1.5 \mathrm{~h}$ of aerobic incubation as a part of normal development. Period of ethanol (control) or bafilomycin treatment is indicated by a hatched bar, and anoxic treatment is indicated by a solid bar. Spectra used for plotting bafilomycin treatment are selectively displayed for (B) $1 \mathrm{~h}$ of aerobic development, followed by (C) $1 \mathrm{~h}$ of anoxia-induced quiescence and (D) $2 \mathrm{~h}$ of subsequent aerobic recovery. Control spectra are not shown. All shaded boxes are identical, and serve to emphasize changes in chemical shift and shape of the $\mathrm{P}_{\mathrm{i}}$ peaks.
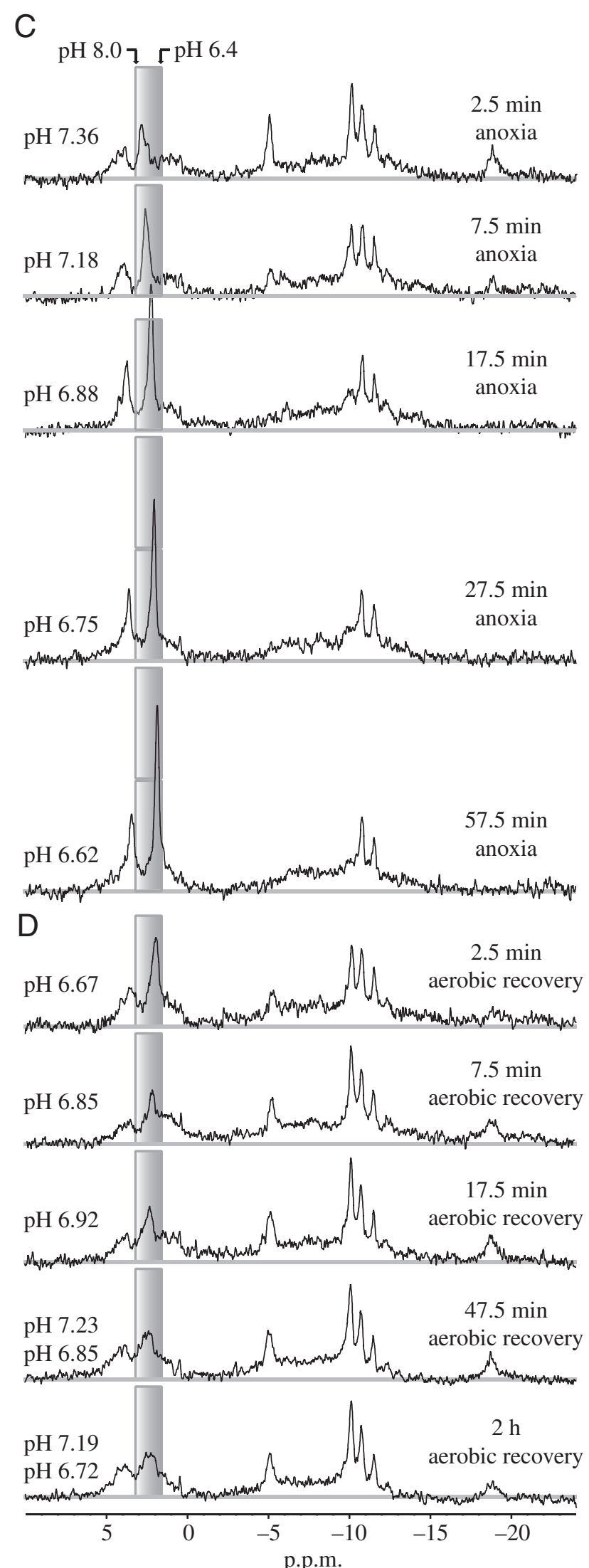


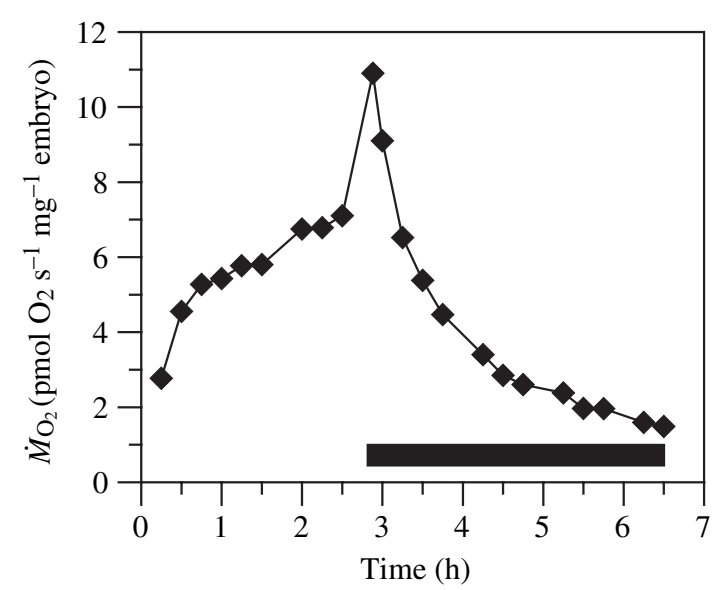

Fig. 5. Effect of $50 \mu \mathrm{mol} \mathrm{l}^{-1} \mathrm{CCCP}$ on oxygen consumption $\left(\dot{M}_{\mathrm{O}_{2}}\right)$ by dechorionated A. franciscana embryos. Embryos were allowed to develop for $2.75 \mathrm{~h}$ before the addition of the protonophore CCCP. Period of incubation with CCCP is indicated by a solid bar.

concentrations of this V-ATPase inhibitor arrests development of the anoxia-tolerant gastrula. The primary goals of the current study were to determine whether the effects of bafilomycin are linked to intracellular acidification via the dissipation of proton gradients normally maintained by the VATPase during aerobic development and, if so, to determine whether such dissipation is involved in the acidification observed under anoxia. For intact dechorionated embryos, we succeeded in demonstrating that (1) prolonged incubation with bafilomycin produces a moderate but reversible intracellular acidification, (2) exposure to bafilomycin does not affect the capacity for acidification under anoxia, (3) prolonged exposure to bafilomycin precludes recovery from acidification induced by anoxia and (4) the lipid-soluble protonophore CCCP also penetrates the embryonic cuticle and produces an acidification similar to that occurring after $1 \mathrm{~h}$ of anoxia. Together, these data confirm the presence of functional V-ATPase during the anoxia-tolerant stage of development, provide direct evidence of the importance of this proton pump during recovery from anoxia and suggest that global dissipation of proton gradients occurs during the first hour of exposure to anoxia.

The ${ }^{31} \mathrm{P}-\mathrm{NMR}$ data presented here suggest that the sequestration of protons in compartment(s) with low $\mathrm{P}_{\mathrm{i}}$ content is the major contributor to intracellular alkalinization during aerobic recovery from anoxia. Following $1 \mathrm{~h}$ of anoxia in the presence of $4 \mu \mathrm{mol} \mathrm{l}^{-1}$ bafilomycin, NTP chemical shifts reappear less than $30 \mathrm{~min}$ after the restoration of oxygenated conditions. This is consistent with the restoration of oxidative phosphorylation. During the same period, however, only a 0.3 unit alkalinization of pHi (6.6-6.9) is observed in embryos sufficiently loaded with bafilomycin (Fig. 4). Using these data, and assuming that buffering capacity is relatively constant between $\mathrm{pH} 6.7$ and 7.7, one can estimate that oxidative phosphorylation and the resynthesis of ATP combined could only account for $\sim 30 \%$ of the protons required for the full recovery of pHi after $1 \mathrm{~h}$ of anoxia. This value is at the lower
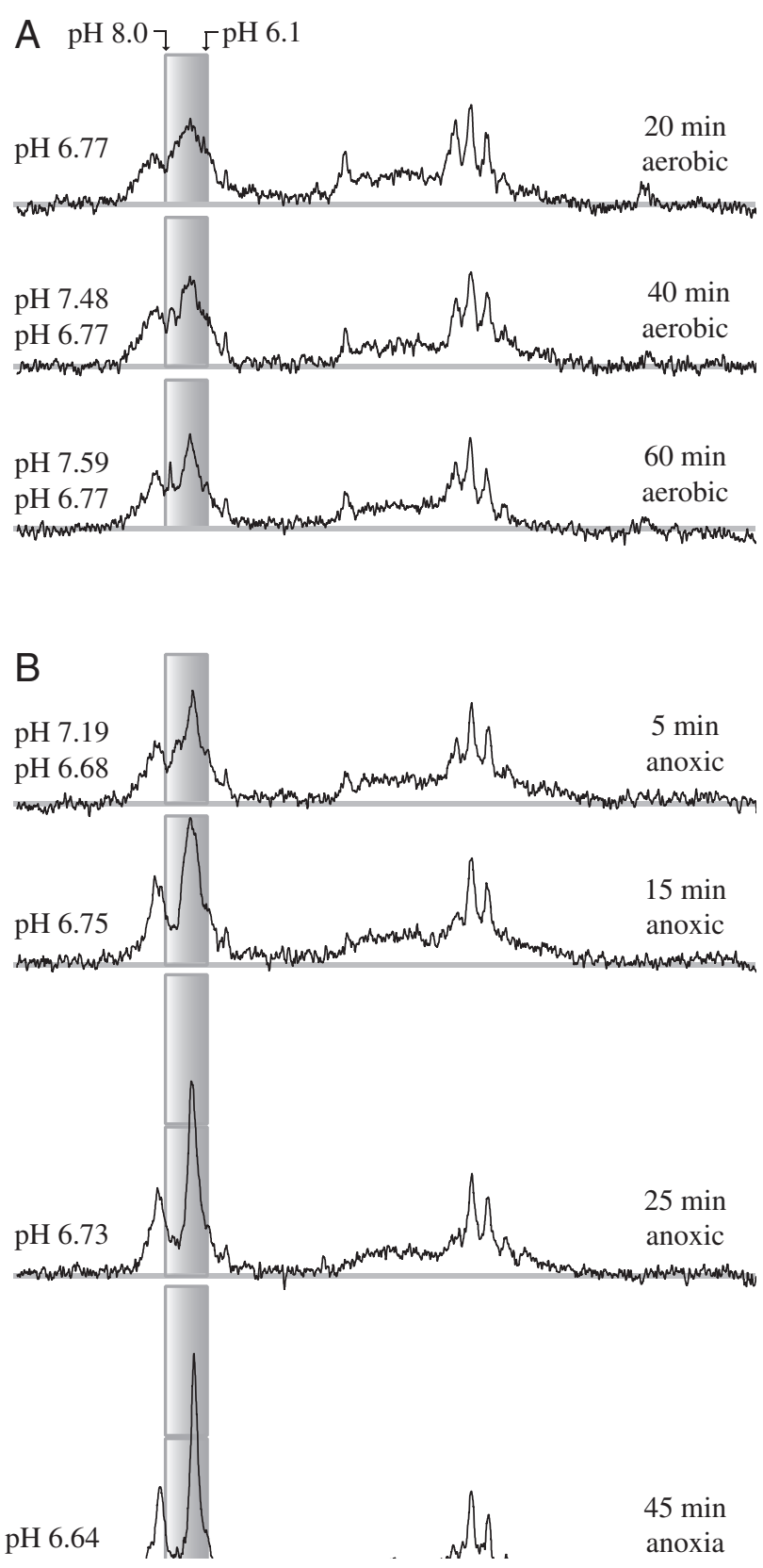

Fig. 6. Representative ${ }^{31} \mathrm{P}-\mathrm{NMR}$ spectra for long-term treatment of dechorionated embryos with CCCP. Embryos were incubated for $30 \mathrm{~h}$ on ice in a sterile-filtered solution of $0.25 \mathrm{~mol} \mathrm{l}^{-1} \mathrm{NaCl}$ and $20 \mu \mathrm{mol} \mathrm{l}^{-1}$ CCCP. ${ }^{31} \mathrm{P}-\mathrm{NMR}$ spectra were subsequently acquired during $1 \mathrm{~h}$ of superfusion with CCCP under (A) aerobic conditions, (B) followed by anoxia.

end of the range estimated by Kwast et al. (1995). Their calculations suggest that oxidative phosphorylation, under acidic conditions known to inhibit carbohydrate metabolism in A. franciscana embryos, could account for $13-45 \%$ of the proton consumption required to shift $\mathrm{pHi}$ from the anoxic value of 6.7 to the aerobic value of 7.7, while the resynthesis of NTP could contribute another 13-27\% over the same $\mathrm{pH}$ range. Our data contribute to this work by demonstrating that the VATPase is active at a $\mathrm{pH}$ of $\leqslant 6.9$ and that proton pumping by 


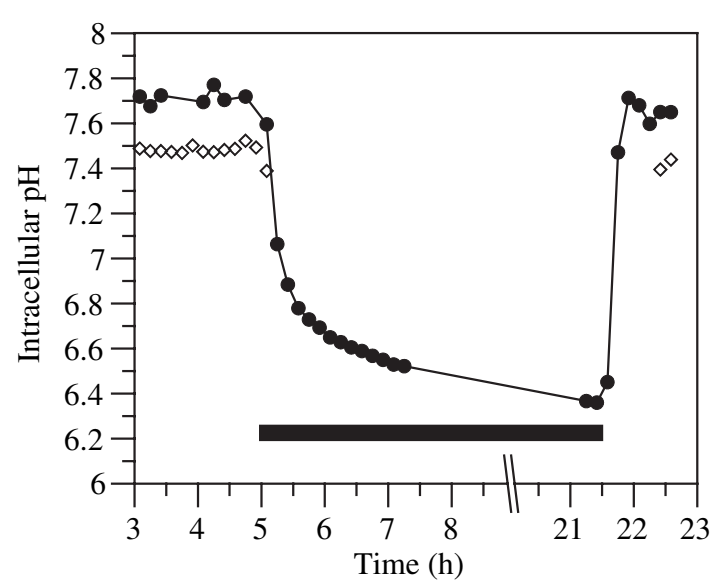

Fig. 7. Intracellular $\mathrm{pH}$ status for dechorionated A. franciscana embryos during exposure to, and recovery from, $16.5 \mathrm{~h}$ of anoxia. Intracellular $\mathrm{pH}$ is plotted as a function of time for two $\mathrm{P}_{\mathrm{i}}$ chemical shifts identified during early development for aseptically treated embryos superfused with $0.25 \mathrm{~mol} \mathrm{l}^{-1} \mathrm{NaCl}$. Solid circles indicate $\mathrm{pH}$ determined from main downfield (alkaline) $\mathrm{P}_{\mathrm{i}}$ resonance, and open diamonds indicate the upfield (acidic) $\mathrm{P}_{\mathrm{i}}$ resonance. Period of anoxic treatment is indicated by a solid bar. Treatment conditions used mimic those employed in the anoxia studies of Kwast et al. (1995).

this enzyme is responsible for a large fraction of the alkalinization occurring during aerobic recovery following $1 \mathrm{~h}$ of anoxia.

Recovery from intracellular acidification in A. franciscana embryos is more likely to involve sequestration of protons in intracellular compartments than secretion of protons across the plasma membrane. While the V-ATPase has been observed to function in proton secretion in the gas gland cells of eel swimbladder when $\mathrm{pH}_{\mathrm{i}}$ is low (Sotz et al., 2002), this recovery mechanism would be less effective in A. franciscana embryos because the extracellular compartment is spatially constrained by a cuticle and likely to have a buffering capacity equal to or less than the cytosol. Thus, although it is possible that protons are extruded to the extracellular space during recovery from the intracellular acidification induced by anoxia, it seems more probable that alkalinization of $\mathrm{pH}_{\mathrm{i}}$ would involve the sequestration of protons within intracellular compartments. These compartments would require a $\mathrm{P}_{\mathrm{i}}$ content low enough relative to the cytosol to make their visualization with ${ }^{31} \mathrm{P}-\mathrm{NMR}$ difficult. In this way, the protons used to acidify the main intracellular compartment under anoxia would effectively be masked during recovery and aerobic development. As mentioned earlier, compartments involved in this event could include lysosomes, transport vesicles, Golgi apparatus and yolk platelets. It seems unlikely that such organelles would 'hyper acidify' at a time when metabolic processes are being restarted, because their functional capacity could be adversely affected. It is more probable that they serve as proton reservoirs that are emptied upon exposure to anoxia and refilled during aerobic recovery. This would suggest that dissipation of proton gradients under anoxia contributes to the observed intracellular acidification.
While incubation with bafilomycin did produce a slight acidification of the intracellular space under aerobic conditions, the transient nature of this acidification suggests that V-ATPase inhibition alone is not enough to induce a rapid dissipation of proton gradients. One might speculate that inactivation of proton pumping would be coordinated with the opening/activation of dissipative paths if proton gradients collapse under anoxia. Opening of dissipative channels might be triggered directly by oxygen removal; mechanisms of oxygen sensing is an exciting area of current research in many organisms including A. franciscana embryos (Kwast and Hand, 1996).

Even if the aerobic embryos are able to maintain $\mathrm{pH}_{\mathrm{i}}$ in the absence of V-ATPase activity, inhibition of this enzyme is still likely to affect development given its broad functional repertoire. Such is the case for Xenopus, in which bafilomycin treatment appears to block both yolk platelet degradation and cellular differentiation (Fagotto and Maxfield, 1994). These results are consistent with our observations, which demonstrate that the development of brine shrimp embryos is arrested by incubation in low concentrations of bafilomycin (Covi and Hand, 2005), despite an ability to maintain $\mathrm{pH}_{\mathrm{i}}$ and NTP status for at least $5 \mathrm{~h}$ during exposure to this inhibitor. One potential explanation would be an inability to initiate the first round of lysosome-mediated yolk degradation, which peaks at the start of emergence (Perona et al., 1988; Perona and Vallejo, 1989). This possibility is supported by the observation that both yolk degradation and development can be reversibly inhibited in Artemia embryos by incubation with lysosomotrophic agents (Perona et al., 1987).

Importantly, ${ }^{31} \mathrm{P}-\mathrm{NMR}$ experiments using CCCP support previous theoretical calculations, which suggested that the dissipation of proton concentration gradients will have a large impact on $\mathrm{pH}_{\mathrm{i}}$ of encysted embryos. We estimated that the dissipation of proton gradients across the membranes of Golgi complex, endosomal and exocytotic vesicles, lysosomes and yolk platelets in A. franciscana embryos could release enough protons to account for $50 \%$ of the acidification occurring during the first hour of exposure to anoxia (Covi and Hand, 2005). Here, we directly tested the effect of proton gradient dissipation with the protonophore CCCP. If a global dissipation of proton gradients contributes to $\mathrm{pH}_{\mathrm{i}}$ acidification under anoxia, then exposure of aerobic embryos to CCCP should induce a $\mathrm{pH}_{\mathrm{i}}$ similar to that observed in anoxic embryos. This acidification would be produced by both a net hydrolysis of ATP - due to an uncoupling of oxidative phosphorylation and a dissipation of proton concentration gradients. In addition, subsequent exposure of CCCP-treated embryos to anoxia should not cause further acidification of the already acidic $\mathrm{pH}_{\mathrm{i}}$ produced by CCCP exposure. These responses are in fact exactly what we observed in whole embryos incubated with this protonophore (Fig. 6). Thus, it appears that global proton gradient dissipation occurs under anoxia, and this event contributes to the acidification observed in quiescent embryos.

There is a mismatch between the kinetics of acidification under anoxia and alkalinization upon reoxygenation, which is 
consistent with the involvement of proton gradient dissipation in the acidification event. Under anoxia, $\mathrm{pH}_{\mathrm{i}}$ declines approximately 0.8 units during the first $22.5 \mathrm{~min}$ of exposure (Busa et al., 1982; Kwast et al., 1995). This rapid drop is followed by a slower phase of acidification during which $\mathrm{pH}_{\mathrm{i}}$ decreases another 0.2 units over a period of $30 \mathrm{~min}(57.5 \mathrm{~min}$ total anoxia exposure). Busa et al. (1982) observed that this slow phase of acidification continues overnight, eventually reaching a $\mathrm{pH}$ of 6.3 ( $\geqslant 1.4$ unit total acidification). Our results support this finding, as we observed a $\mathrm{pH}$ of 6.36 in embryos incubated for $16.5 \mathrm{~h}$ under anoxia, and 6.25 after $30 \mathrm{~h}$ of exposure. However, unlike the acidification occurring under anoxia, alkalinization of $\mathrm{pH}_{\mathrm{i}}$ during recovery is remarkably fast. Kwast et al. (1995) demonstrated that $\mathrm{pH}_{\mathrm{i}}$ is completely restored within the first $22.5 \mathrm{~min}$ of reoxygenation following $2 \mathrm{~h}$ of anoxia. Our work extends this finding by demonstrating a 1.35 unit alkalinization during only $25 \mathrm{~min}$ of aerobic recovery following $16.5 \mathrm{~h}$ of anoxia (Fig. 7). One potential explanation for the disparity in kinetics between the two transitions could be that proton gradient dissipation under anoxia is slow relative to the resequestration of protons by active transport during recovery.

In conclusion, the data presented here demonstrate that the dissipation of proton gradients under anoxia helps to produce an intracellular acidification that is critical to the induction of quiescence in encysted embryos of the brine shrimp, Artemia franciscana. Taking into account changes in the metabolic state of the embryo, we constructed a schematic model of the role played by proton chemical gradients during the induction of, and recovery from, anoxia-induced quiescence (Fig. 8). In this model, exposure to anoxia induces a drop in cellular ATP as oxidative phosphorylation is arrested. Within the first $5 \mathrm{~min}$ of exposure to anoxia, this leads to a cessation of proton transport by pumps such as the V-ATPase and an activation of proton dissipative paths. The combined result is a 1 unit drop in intracellular $\mathrm{pH}$ over approximately $1 \mathrm{~h}$. The return of oxidative phosphorylation upon reoxygenation of the embryos causes ATP levels to rise, which in turn activates the V-ATPase. Subsequent proton pumping into the intracellular compartment causes further alkalinization of $\mathrm{pH}_{\mathrm{i}}$, which in turn helps to facilitate the resumption of development and metabolic processes inhibited by low $\mathrm{pH}$. Importantly, this novel mechanism for regulated acidification/alkalinization of the intracellular space may represent a critical adaptive trait conferring extreme anoxia tolerance to this species. Such an adaptive trait could answer the question of what sets the anoxia tolerance of Artemia embryos apart from that of animals such as goldfish and turtles, which was recently posed by Guppy and Withers (1999).

This study was supported by DARPA grant N00173-01-1G011 and NIH grant 1-R01-GM071345-01. We would like to

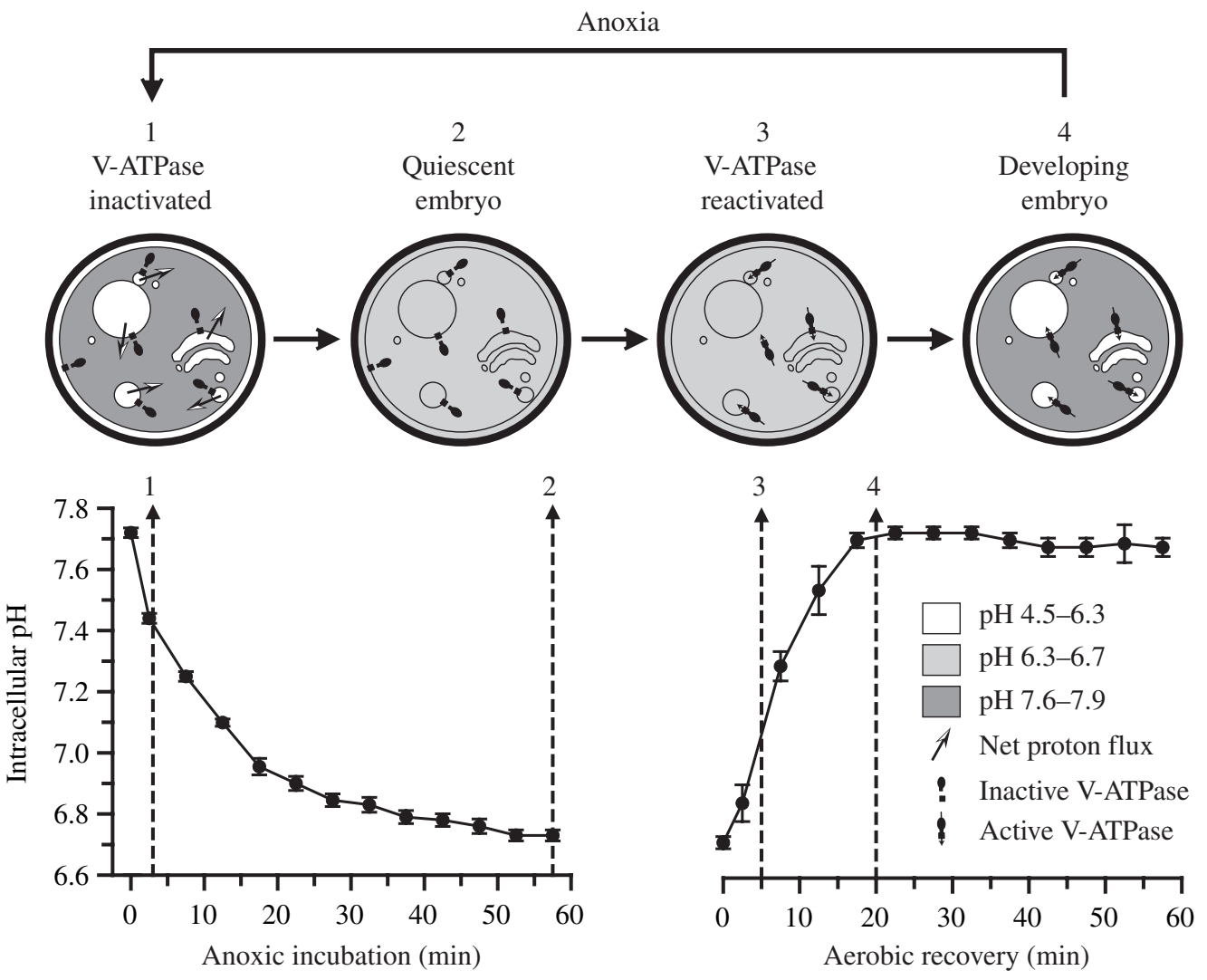

Fig. 8. Schematic representation for the role of proton concentration gradients in intracellular pH shifts induced by transitions between anoxic and aerobic states in encysted embryos of the brine shrimp, Artemia franciscana. Plots were modified from Kwast et al. (1995), and the data were derived from ${ }^{31} \mathrm{P}-\mathrm{NMR}$ estimates of intracellular $\mathrm{pH}$. Broken lines indicate time points corresponding to the embryo diagrams above and are numbered accordingly. 
thank Dr Helmut Wieczorek and Dr Markus Huss for useful discourse regarding the incubation of Artemia franciscana embryos with bafilomycin. We would also like to thank Dr James Moroney for comments regarding experimental design and the generous provision of CCCP for this study.

\section{References}

Abreu, L. A., Valle, D., Manso, P. P., Facanha, A. R., Pelajo-Machado, M., Masuda, H., Masuda, A., Vaz, I., Jr, Lenzi, H., Oliveira, P. L. et al. (2004). Proteolytic activity of Boophilus microplus Yolk pro-Cathepsin D (BYC) is coincident with cortical acidification during embryogenesis. Insect Biochem. Mol. Biol. 34, 443-449.

Anchordoguy, T. J. and Hand, S. C. (1994). Acute blockage of the ubiquitinmediated proteolytic pathway during invertebrate quiescence. Am. $J$. Physiol. 267, R895-R900.

Busa, W. B. and Nuccitelli, R. (1984). Metabolic regulation via intracellular pH. Am. J. Physiol. 246, R409-R438.

Busa, W. B., Crowe, J. H. and Matson, G. B. (1982). Intracellular pH and the metabolic status of dormant and developing Artemia embryos. Arch. Biochem. Biophys. 216, 711-718.

Clegg, J. (1997). Embryos of Artemia franciscana survive four years of continuous anoxia: the case for complete metabolic rate depression. J. Exp. Biol. 200, 467-475.

Clegg, J. S. and Conte, F. P. (1980). Cellular and developmental biology of Artemia. In The Brine Shrimp Artemia. Vol. 2 (ed. G. Persoone, P. Sorgeloos, O. Roels and E. Jaspers), pp. 83-103. Whitteren, Belgium: Universa Press.

Clegg, J. S., Jackson, S. A. and Popov, V. I. (2000). Long-term anoxia in encysted embryos of the crustacean, Artemia franciscana: viability, ultrastructure, and stress proteins. Cell Tissue Res. 301, 433-446.

Conte, F. P., Droukas, P. C. and Ewing, R. D. (1977). Development of sodium regulation and De Novo synthesis of $\mathrm{Na}^{+} \mathrm{K}$-activated ATPase in larval brine shrimp, Artemia salina. Exp. Zool. 202, 339-362.

Covi, J. A. and Hand, S. C. (2005). V-ATPase expression during development of Artemia franciscana embryos: potential role for proton gradients in anoxia signaling. J. Exp. Biol. 208, 2783-2798.

Drinkwater, L. E. and Clegg, J. S. (1991). Experimental biology of cyst diapause. In Artemia Biology (ed. R. A. Browne, P. Sorgeloose and C. N. A. Trotman), pp. 93-117. Boca Raton: CRC Press.

Drose, S. and Altendorf, K. (1997). Bafilomycins and concanamycins as inhibitors of V-ATPases and P-ATPases. J. Exp. Biol. 200, 1-8.

Eads, B. D. and Hand, S. C. (2003). Mitochondrial mRNA stability and polyadenylation during anoxia-induced quiescence in the brine shrimp Artemia franciscana. J. Exp. Biol. 206, 3681-3692.

Fagotto, F. (1995). Regulation of yolk degradation, or how to make sleepy lysosomes. J. Cell Sci. 108, 3645-3647.

Fagotto, F. and Maxfield, F. R. (1994). Changes in yolk platelet pH during Xenopus laevis development correlate with yolk utilization. A quantitative confocal microscopy study. J. Cell Sci. 107, 3325-3337.

Forgac, M. (2000). Structure, mechanism and regulation of the clathrin-coated vesicle and yeast vacuolar H(+)-ATPases. J. Exp. Biol. 203, 71-80.

Futai, M., Oka, T., Sun-Wada, G., Moriyama, Y., Kanazawa, H. and Wada, Y. (2000). Luminal acidification of diverse organelles by V-ATPase in animal cells. J. Exp. Biol. 203, 107-116.

Guppy, M. and Withers, P. (1999). Metabolic depression in animals: physiological perspectives and biochemical generalizations. Biol. Rev. 74, $1-40$.
Hand, S. C. (1998). Quiescence in Artemia franciscana embryos: reversible arrest of metabolism and gene expression at low oxygen levels. J. Exp. Biol. 201, 1233-1242.

Huss, M. and Wieczorek, H. (2003). The reversible dissociation of V-ATPase is influenced by nucleotides. Comp. Biochem. Physiol. 134, 18.P2.

Kane, P. M. and Smardon, A. M. (2003). Assembly and regulation of the yeast vacuolar $\mathrm{H}^{+}$-ATPase. J. Bioenerg. Biomembr. 35, 313-321.

Kettner, C., Obermeyer, G. and Bertl, A. (2003). Inhibition of the yeast Vtype ATPase by cytosolic ADP. FEBS Lett. 535, 119-214.

Kwast, K. E. and Hand, S. C. (1993). Regulatory features of protein synthesis in isolated mitochondria from Artemia embryos. Am. J. Physiol. 265, R1238-R1246.

Kwast, K. E. and Hand, S. C. (1996). Acute depression of mitochondrial protein synthesis during anoxia: contributions of oxygen sensing, matrix acidification, and redox state. J. Biol. Chem. 271, 7313-7319.

Kwast, K. E., Shapiro, J. I., Rees, B. B. and Hand, S. C. (1995). Oxidative phosphorylation and the realkalinization of intracellular $\mathrm{pH}$ during recovery from anoxia in Artemia franciscana embryos. Biochim. Biophys. Acta 1232, 5-12.

Menze, M. A., Hutchinson, K., Laborde, S. M. and Hand, S. C. (2005). Mitochondrial permeability transition in the crustacean Artemia franciscana: Absence of a $\mathrm{Ca}^{2+}$-regulated pore in the face of profound calcium storage. Am. J. Physiol. 289, R68-R76.

Motta, L. S., da Silva, W. S., Oliveira, D. M., de Souza, W. and Machado, E. A. (2004). A new model for proton pumping in animal cells: the role of pyrophosphate. Insect Biochem Mol. Biol. 34, 19-27.

Nishi, T. and Forgac, M. (2002). The vacuolar (H+)-ATPases - nature's most versatile proton pumps. Nat. Rev. Mol. Cell Biol. 3, 94-103.

Perona, R. and Vallejo, C. G. (1989). Mechanisms of yolk degradation in Artemia - a morphological study. Comp. Biochem. Physiol. 94, 231-242.

Perona, R., Ezquieta, B. and Vallejo, C. G. (1987). The degradation of yolk in Artemia. In Artemia Research and Its Applications. Vol. 2 (ed. W. Decleir, L. Moens, H. Slegers, E. Jaspers and P. Sorgeloos), pp. 105-123. Wetteren, Belgium: Universa Press.

Perona, R., Bes, J. C. and Vallejo, C. G. (1988). Degradation of yolk in the brine shrimp Artemia. Biochemical and morphological studies on the involvement of the lysosomal system. Biol. Cell 63, 361-366.

Price, S. (1967). Dimethyl sulfoxide: failure to penetrate encysted Artemia embryos. Proc. Soc. Exp. Biol. Med. 126, 398-399.

Sotz, E., Niederstatter, H. and Pelster, B. (2002). Determinants of intracellular $\mathrm{pH}$ in gas gland cells of the swimbladder of the European eel Anguilla anguilla. J. Exp. Biol. 205, 1069-1075.

Stocco, D. M., Beers, P. C. and Warner, A. H. (1972). Effect of anoxia on nucleotide metabolism in encysted embryos of the brine shrimp. Dev. Biol. 27, 479-493.

Trotman, C. N. A. (1991). Normality and abnormality in early development. In Artemia Biology (ed. R. A. Brown, P. Sorgeloos and C. N. A. Trotman), pp. 75-92. Boca Raton: CRC Press.

Warner, A. H. and Clegg, J. S. (2001). Diguanosine nucleotide metabolism and the survival of artemia embryos during years of continuous anoxia. Eur. J. Biochem. 268, 1568-1576.

Warner, A. H., Chu, P. P., Shaw, M. F. and Criel, G. (2002). Yolk platelets in artemia embryos: are they really storage sites of immature mitochondria? Comp. Biochem. Physiol. 132B, 491-503.

Wieczorek, H., Huss, M., Merzendorfer, H., Reineke, S., Vitavska, O. and Zeiske, W. (2003). The insect plasma membrane H+ V-ATPase: intra-, inter-, and supramolecular aspects. J. Bioenerg. Biomembr. 35, 359-366.

Willsie, J. K. and Clegg, J. S. (2002). Small heat shock protein p26 associates with nuclear lamins and HSP70 in nuclei and nuclear matrix fractions from stressed cells. J. Cell Biochem. 84, 601-614. 